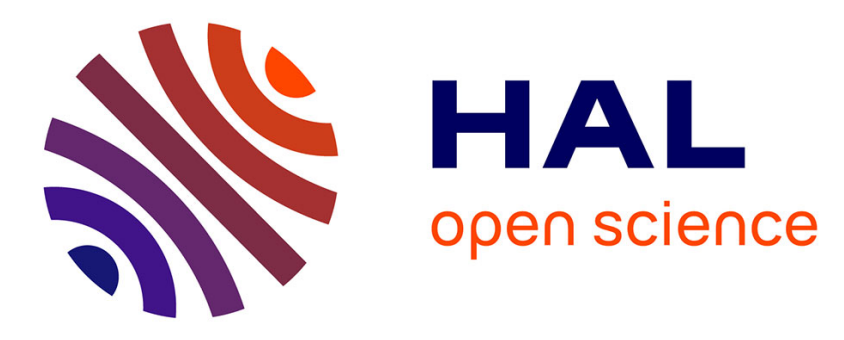

\title{
Insights into Single-Molecule-Magnet Behavior from the Experimental Electron Density of Linear Two-Coordinate Iron Complexes
}

Maja Thomsen, Andreas Nyvang, James Walsh, Philip Bunting, Jeffrey Long, Frank Neese, Michael Atanasov, Alessandro Genoni, Jacob Overgaard

\section{- To cite this version:}

Maja Thomsen, Andreas Nyvang, James Walsh, Philip Bunting, Jeffrey Long, et al.. Insights into Single-Molecule-Magnet Behavior from the Experimental Electron Density of Linear Two-Coordinate Iron Complexes. Inorganic Chemistry, 2019, 58 (5), pp.3211-3218. 10.1021/acs.inorgchem.8b03301 . hal-02196490

\section{HAL Id: hal-02196490 \\ https://hal.univ-lorraine.fr/hal-02196490}

Submitted on 29 May 2020

HAL is a multi-disciplinary open access archive for the deposit and dissemination of scientific research documents, whether they are published or not. The documents may come from teaching and research institutions in France or abroad, or from public or private research centers.
L'archive ouverte pluridisciplinaire HAL, est destinée au dépôt et à la diffusion de documents scientifiques de niveau recherche, publiés ou non, émanant des établissements d'enseignement et de recherche français ou étrangers, des laboratoires publics ou privés. 


\section{Insights into single-molecule magnet behavior from}

\section{the experimental electron density of linear two-}

\section{coordinate iron complexes}

Maja K. Thomsen, ${ }^{1}$ Andreas Nyvang, ${ }^{1}$ James P. S. Walsh ${ }^{2}$, Philip C. Bunting, ${ }^{3}$ Jeffrey R. Long ${ }^{3,4,5}$, Frank Neese ${ }^{*, 6}$, Michael Atanasov ${ }^{* 6,7}$, Alessandro Genoni, ${ }^{8}$ Jacob Overgaard $^{*}$

${ }^{1}$ Department of Chemistry \& Centre for Materials Crystallography, Aarhus University, DK-8000 Aarhus C, Denmark

${ }^{2}$ Department of Chemistry, Northwestern University, 2145 Sheridan Rd, Evanston, IL 60208 USA

${ }^{3}$ Department of Chemistry, University of California, Berkeley, California 94720-1460, USA

${ }^{4}$ Department of Chemical and Biomolecular Engineering, University of California, Berkeley, California 94720-1460, USA

${ }^{5}$ Materials Sciences Division, Lawrence Berkeley National Laboratory, Berkeley, California 94720, USA

${ }^{6}$ Max Planck Institute for Chemical Energy Conversion, Stiftstraße 34-36, Mülheim an der Ruhr, D-45470, Germany 
${ }^{7}$ Institute of General and Inorganic Chemistry, Bulgarian Academy of Sciences, Sofia 1113, Bulgaria

${ }^{8}$ Université de Lorraine and CNRS, Laboratoire de Physique et Chimie Théoriques (LPCT), UMR CNRS 7019, 1 Boulevard Arago, F-57078 Metz, France.

Keywords: Single molecule magnets, synchrotron X-ray diffraction, electron densities, ab initio calculations.

\begin{abstract}
A breakthrough in the study of single-molecule magnets occurred with the discovery of zerofield slow magnetic relaxation and hysteresis for the linear iron(I) complex $\left[\mathrm{Fe}\left(\mathrm{C}\left(\mathrm{SiMe}_{3}\right)_{3}\right)_{2}\right]^{-}(\mathbf{1})$, which has one of the largest spin-reversal barriers among mononuclear transition metal singlemolecule magnets. Theoretical studies have suggested that the magnetic anisotropy in $\mathbf{1}$ is made possible by pronounced stabilization of the iron $\mathrm{d}_{\mathrm{z}^{2}}$ orbital due to $3 \mathrm{~d}_{\mathrm{z}^{2}}-4 \mathrm{~s}$ mixing, an effect which is predicted to be less pronounced in the neutral iron(II) complex $\mathrm{Fe}\left(\mathrm{C}\left(\mathrm{SiMe}_{3}\right)_{3}\right)_{2}$ (2). However, experimental support for this interpretation has remained lacking. Here, we use high-resolution single-crystal X-ray diffraction data to generate multipole models of the electron density in these two complexes, which clearly show that the iron $\mathrm{d}_{\mathrm{z}^{2}}$ orbital is more populated in $\mathbf{1}$ than in $\mathbf{2}$. This result can be interpreted as arising from greater stabilization of the $d_{z^{2}}$ orbital in $\mathbf{1}$, thus offering an unprecedented experimental rationale for the origin of magnetic anisotropy in $\left[\mathrm{Fe}\left(\mathrm{C}\left(\mathrm{SiMe}_{3}\right)_{3}\right)_{2}\right]^{-}$
\end{abstract}

\title{
Introduction
}


Since the discovery of magnetic blocking in a dodecanuclear manganese cluster more than two decades ago, ${ }^{1}$ the search for single-molecule magnets with improved operating temperatures has burgeoned beyond the study of transition metal clusters to encompass complexes of the lanthanides ${ }^{2}$ and actinides $^{3}$ as well as mononuclear transition metal compounds. ${ }^{4-7}$ Arguably, the original impetus for this expansion arose both from the discovery of slow magnetic relaxation and magnetic hysteresis in mononuclear lanthanide complexes ${ }^{8}$ and the realization that magnetic anisotropy-more than spin-is the key factor in dictating slow magnetic relaxation for transition metal compounds. ${ }^{9-10}$ Indeed, contemporary efforts in the design of single-molecule magnets are focused largely on various strategies to exploit and enhance magnetic anisotropy toward the realization of ever larger energy barriers to spin relaxation and high-temperature magnetic hysteresis. ${ }^{11-13}$ Magnetic anisotropy is a phenomenon that derives ultimately from the presence of non-zero orbital angular momentum in the electronic ground state of a magnetic ion, which itself results either from the presence of (quasi)-degenerate electronic configurations (a first-order effect) or via mixing with excited electronic states possessing non-zero orbital angular momentum (a second order effect). Coupling of the spin and orbital angular momentum removes the degeneracy of the ground state and creates the observed anisotropy, which can also be enhanced by the ligand field.

The magnitude of the spin-orbit coupling increases with atomic number, and can reach maximal values with heavy elements such as the lanthanides and actinides. Many trivalent lanthanide ions in particular exhibit both a large spin and unquenched orbital angular momentum that arise from the near degeneracy of the well-shielded $4 \mathrm{f}$ orbitals, and thus perhaps unsurprisingly the pursuit of lanthanide-based single-molecule magnets represents one of the most promising avenues to-date. Indeed, lanthanide compounds have set recent records for both 
magnetic relaxation barrier and hysteresis temperature. ${ }^{11,13}$ Various levels of theory have been developed to predict the magnitude and orientation of the magnetic anisotropy in lanthanide systems, and are in some cases quite successful ${ }^{14}$ while experimentally the anisotropy can be quantified using neutron diffraction. ${ }^{15}$

For the transition metal ions, first-order orbital angular momentum is typically quenched by the ligand field, but a second-order effect can arise when the absolute value of the d-orbital splitting is optimized such that excited states exhibiting orbital angular momentum are close in energy to the ground state. Alternatively, a strategy to achieve first-order orbital angular momentum in mononuclear transition metal complexes is to target linear coordination environments, which ensure the presence of degenerate orbitals that will be unaffected by JahnTeller distortions. This approach was first realized in the development of a series of linear, twocoordinate high-spin iron(II) single-molecule magnets ${ }^{8}$, including the complex $\mathrm{Fe}\left(\mathrm{C}\left(\mathrm{SiMe}_{3}\right)_{3}\right)_{2}{ }^{6}$, ${ }^{16-21}$ However, an applied magnetic field was required in order to observe slow magnetic relaxation for these complexes, due at least in part to pronounced ground-state quantum tunneling of the magnetization associated with the non-Kramers-integer spin-iron(II) ion. ${ }^{6}$ Notably, successful chemical reduction of $\mathrm{Fe}\left(\mathrm{C}\left(\mathrm{SiMe}_{3}\right)_{3}\right)_{2}$ was later achieved to generate the linear, two-coordinate compound $[\mathrm{K}(\mathrm{crypt}-222)]\left[\mathrm{Fe}\left(\mathrm{C}\left(\mathrm{SiMe}_{3}\right)_{3}\right)_{2}\right](\mathbf{1}),{ }^{22}$ with a half-integer spin iron(I) center. Compound $\mathbf{1}$ behaves as a single-molecule magnet under zero dc field and exhibits a relaxation barrier ${ }^{23,24}$ of $246(3) \mathrm{cm}^{-1}$ as well as magnetic hysteresis to $6 \mathrm{~K}$.

These results can be successfully interpreted within a ligand field model. In a strictly cylindrically symmetric coordination environment (i.e., strictly axial symmetry, with the z-axis oriented along the axial direction), the d-orbitals split into $\left(\mathrm{d}_{\mathrm{x}^{2}-\mathrm{y}^{2}}, \mathrm{~d}_{\mathrm{xy}}\right),\left(\mathrm{d}_{\mathrm{xz}}, \mathrm{d}_{\mathrm{yz}}\right)$, and $\left(\mathrm{d}_{\mathrm{z}^{2}}\right)$ sets. In the limit of purely electrostatic crystal field theory, the d-orbitals are split depending on the 
projection of the angular momentum on the z-axis $\left(m_{l}\right)$. Larger values of $m_{l}$ are associated with less repulsion due to the axial ligands, and thus these orbitals are lowest in energy. However, symmetry-allowed $3 \mathrm{~d}_{\mathrm{z}^{2}}-4 \mathrm{~s}$ orbital mixing may lower the energy of the molecular orbital of $\mathrm{d}_{\mathrm{z}^{2}}$ parentage below that of the $m_{l}=1$ and $m_{l}=2 \mathrm{~d}$-orbital sets. Assuming electron configurations of $3 d^{7}\left(\right.$ not $\left.3 d^{6} 4 s^{1}\right)$ and $3 d^{6}$ for the iron(I) and iron(II) centers, respectively, ${ }^{25}$ the d-orbital sets for $\mathbf{1}$ and the neutral molecule $\mathrm{Fe}\left(\mathrm{C}\left(\mathrm{SiMe}_{3}\right)_{3}\right)_{2}$ (2) will necessarily require different ordering to achieve ground states with non-zero orbital angular momentum. In particular, a non-zero orbital angular momentum requires an odd electron count in one of the degenerate sets of d-orbitals-i.e., in $\left(\mathrm{d}_{\mathrm{x}^{2}-\mathrm{y}^{2}}, \mathrm{~d}_{\mathrm{xy}}\right)$ or $\left(\mathrm{d}_{\mathrm{xz}}, \mathrm{d}_{\mathrm{yz}}\right)$. For this scenario to occur, the $\mathrm{d}_{\mathrm{z}^{2}}$ orbital in $\mathbf{1}$ must be lowest in energy, while in $\mathbf{2}$ it must either be the highest in energy or in between the $\left(\mathrm{d}_{\mathrm{x}^{2}-\mathrm{y}^{2}}, \mathrm{~d}_{\mathrm{xy}}\right)$ and $\left(\mathrm{d}_{\mathrm{xz}}, \mathrm{d}_{\mathrm{yz}}\right)$ sets.

$A b$ initio ligand field theory ${ }^{26}$ calculations have predicted that the $\mathrm{d}_{\mathrm{z}^{2}}$ orbital is indeed lowest in energy for $\mathbf{1}^{22}$ and of intermediate energy for $\mathbf{2},{ }^{19}$ providing rationale for the reported magnetic relaxation behavior of both compounds ${ }^{22,6}$ However, direct experimental evidence for this unexpected energy ordering of the d-orbitals has remained lacking. Spectroscopic approaches only provide information about the relative energies of the different states, but do not reveal the exact nature of the involved states. In contrast, X-ray diffraction probes the electronic spatial distribution, and it is in principle possible to use this technique to reconstruct a full and complete description of the electron density. Recently, some of us published the first application of this technique to a single-molecule magnet. ${ }^{27}$ Here, we introduce the use of the experimental electron density $(\varrho)$ - derived from accurate high-resolution, low-temperature single crystal Xray diffraction data ${ }^{28}$-to explain the magnetic anisotropy in $\mathbf{1}$ and $\mathbf{2}$. In this work, we have chosen to use a multipole-based reconstruction of the experimental electron density, which allows for unambiguous and direct estimation of d-orbital populations. ${ }^{29}$ 


\section{Experimental Methods}

General Methods. The syntheses of $\mathbf{1}$ and $\mathbf{2}$ were performed as reported in the literature. ${ }^{20,22}$ Single-Crystal X-ray Diffraction. Single crystal X-ray diffraction data for $\mathbf{1}$ were collected using an Oxford Diffraction Supernova instrument equipped with a Mo X-ray source at Aarhus University, an Atlas charge-coupled device detector, and a four-circle goniometer. The crystal was cooled to 100(1) K using an Oxford Cryosystems liquid nitrogen Cryostream device. The data collection consisted of 26 omega scans. The unit cell parameters were determined and the Bragg intensities were integrated using CrysAlisPRO. ${ }^{30}$ The intensities were empirically corrected for absorption using SCALE3 ABSPACK implemented in CrysAlisPRO. ${ }^{30}$ Single crystal X-ray diffraction data for 2 were measured at the beamline BL02B1 at the SPring-8 synchrotron in Japan. The diffractometer at BL02B1 is equipped with a large curved imaging plate detector with automatic readout mounted on a 3-circle goniometer. The crystal was kept in a He gas-stream $(\sim 50 \mathrm{~K})$ and a set of 30 frames was collected. The unit cell parameters were determined and the Bragg intensities were integrated using the software RAPID-AUTO. ${ }^{31}$ An empirical absorption correction was applied. Equivalent reflections were averaged using SORTAV $^{32-33}$ for both $\mathbf{1}$ and $\mathbf{2}$. Further details regarding the data collection and reduction are included in the Supplementary Information. The structures of $\mathbf{1}$ and $\mathbf{2}$ were solved with direct methods using SHELXT ${ }^{34}$ via the Olex2 interface, ${ }^{35}$ and the refinement was carried out by the least squares method using SHELXL-97 ${ }^{36}$ in Olex2. ${ }^{35}$

Electron density analysis. The multipole models of the electron density distributions in $\mathbf{1}$ and $\mathbf{2}$ were refined in XD2006/XD2015 ${ }^{37}$ using the atom-centered Hansen-Coppens formalism. ${ }^{38}$ This model uses density-normalized spherical harmonics, $d_{l m \pm}$, to describe the angular density 
redistribution as shown in equation 1 . The population parameters, $P_{v}$ and $P_{l m \pm}$, and the radial expansion/contraction parameters, $\kappa$ and $\kappa_{l}^{\prime}$, are refined to obtain the final experimental electron density.

$$
\rho_{\text {atom }}(\mathbf{r})=P_{c} \rho_{c}(r)+P_{v} \rho_{v} \kappa^{3}(\kappa r)+\sum_{l=0}^{l_{\max }} \kappa_{l}^{\prime 3} R_{l}(r) \sum_{m=0}^{l} P_{l m \pm} d_{l m \pm}(\theta, \varphi) \quad \text { eq. } 1
$$

The intimate relationship between the spherical harmonic functions used in the multipole model and the hydrogenic orbitals means that it is straightforward to derive experimental d-orbital occupancies by a linear transformation of the multipole population parameters. Further details regarding the multipole modeling for $\mathbf{1}$ and $\mathbf{2}$ are included in the Supplementary Information. A topological analysis $^{39}$ of the multipole model electron density in $\mathbf{1}$ and $\mathbf{2}$ was carried out with the XDPROP module in XD2006. ${ }^{37}$ This module was also used to calculate experimental d-orbital occupancy estimates for the iron atoms in $\mathbf{1}$ and $\mathbf{2}$ from the multipole populations, according to the procedure from Holladay et al. ${ }^{29}$

$\boldsymbol{A} \boldsymbol{b}$ initio calculations. The electronic energy levels as well as relativistic and non-relativistic electron densities of sites Fe1 and Fe2 in $\mathbf{1}$ and the single Fe site in $\mathbf{2}$ were calculated based on the geometries from the crystallographic *.cif files. State average CASSCF calculations on $\mathrm{Fe}^{\mathrm{I}} \mathrm{C}_{2}$ and $\mathrm{Fe}^{\mathrm{II}} \mathrm{C}_{2}$ were performed using the complex geometries from the X-ray cif files. All ten spinfree quartet states of $\mathbf{1}$ (active space consisting of seven electrons distributed over the five $3 \mathrm{~d}$ molecular orbitals, CAS(7,5)) and the five quartet states of $\mathbf{2}$ (active space consisting of six electrons distributed over the five $3 \mathrm{~d}$ molecular orbitals) were taken into account (see the Supplementary Information for input files and selected output results). Inclusion of states of lesser multiplicity (specifically, forty doublets for $\mathbf{1}$ and the 45 triplets and 50 singlets for 2 ) did 
not essentially change the results. Non-relativistic and relativistic (including scalar and spin-orbit effects) densities used in the analysis were read from the correspondingly adjusted "*.gbw" files (adjusted for spin-orbit sublevels of the ${ }^{4} \mathrm{E}$ (1) and ${ }^{5} \mathrm{E}$ (2) states). The relativistic and nonrelativistic densities lead to essentially the same results, supporting the observation that magnetic sublevels for each complex share essentially the same spatial part of the many-electron wavefunctions. All calculations were done with a version of the ORCA program package ${ }^{40-41}$ that was appropriately adjusted for the purpose of the present project developer version.

\section{Results}

Structural results from multipole models. The complex anion of $\mathbf{1}$ and the molecular unit of $\mathbf{2}$ derived from the multipole models are shown in Fig. 1a-c. Surprisingly, the unit cell found for $\mathbf{1}$ is twice as large as that reported previously ${ }^{42}$-although the space group symmetry remains triclinic $P \overline{1}$, the number of independent molecules is doubled such that there are two geometrically distinct $\left[\mathrm{K}(\right.$ crypt-222) $]\left[\mathrm{Fe}\left(\mathrm{C}\left(\mathrm{SiMe}_{3}\right)_{3}\right)_{2}\right]$ units in the asymmetric unit. In each unique complex of $\mathbf{1}$, the $\left[\mathrm{C}\left(\mathrm{SiMe}_{3}\right)_{3}\right]^{-}$ligands are nearly eclipsed, and their relative orientations can be described by the dihedral angle between $\mathrm{Si}-\mathrm{C}-\mathrm{C}^{*}$ and $\mathrm{C}-\mathrm{C}^{*}-\mathrm{Si}^{*}$ planes (the carbon atoms are those bonded to iron), which range from $30.1-30.9^{\circ}$ in $\left[\mathrm{Fe} 1\left(\mathrm{C}\left(\mathrm{SiMe}_{3}\right)_{3}\right)_{2}\right]^{-}$and $14.4-$ $14.8^{\circ}$ in $\left[\mathrm{Fe} 2\left(\mathrm{C}\left(\mathrm{SiMe}_{3}\right)_{3}\right)_{2}\right]^{-}(\mathrm{Fig} .1 \mathrm{~d}, \mathrm{e})$. The average of these ranges is notably close to the average range determined for the previously reported structure $\left(22.6^{\circ}\right.$ versus $21.8-22.2^{\circ}$, respectively $)^{42}$. In compound $\mathbf{2}$, the $\left[\mathrm{C}\left(\mathrm{SiMe}_{3}\right)_{3}\right]^{-}$ligands are in a staggered conformation with much larger dihedral angles in the range from $57.8-61.3^{\circ}$ (Figure 1f). While the $\mathrm{C}-\mathrm{Fe}-\mathrm{C}$ angle in $\mathbf{2}$ is strictly linear due to symmetry (space group $C 2 / c$ ), the $\mathrm{C}-\mathrm{Fe}-\mathrm{C}$ angles in $\mathbf{1}$ are nearly linear at $179.09(3)^{\circ}$ and $179.38(3)^{\circ}$. 
The bond distances in $\mathbf{1}$ and $\mathbf{2}$ are also distinct, highlighting the different iron formal oxidation states in each complex - in $\left[\mathrm{Fe}\left(\mathrm{C}\left(\mathrm{SiMe}_{3}\right)_{3}\right)_{2}\right]^{-}$, the iron formal oxidation state is +1 and the $\mathrm{Fe}-\mathrm{C}$ distances range from 2.0610(7)-2.0653(7) $\AA$, whereas the iron formal oxidation state in $\mathrm{Fe}\left(\mathrm{C}\left(\mathrm{SiMe}_{3}\right)_{3}\right)_{2}$ is +2 and the $\mathrm{Fe}-\mathrm{C}$ distance is $2.0535(4) \AA$. Integration of the experimental multipole model electron densities within atomic basins derived from Bader's Quantum Theory of Atoms in Molecules ${ }^{39}$ afforded iron atomic charges of approximately +0.6 and +1.4 in $\mathbf{1}$ and $\mathbf{2}$, respectively. These values confirm that iron is more positive in $\mathbf{2}$ than in $\mathbf{1}$, as expected, although they are smaller than the formal iron oxidations states in each complex. Similar discrepancies between calculated atomic charge and formal oxidation state have been reported for studies of metal-metal and iron-sulfur bonding ${ }^{43-45}$ and have been attributed to covalent effects that are unaccounted for in a formal atomic charge description.

a

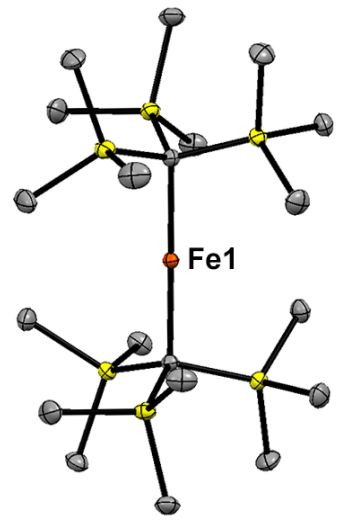

d

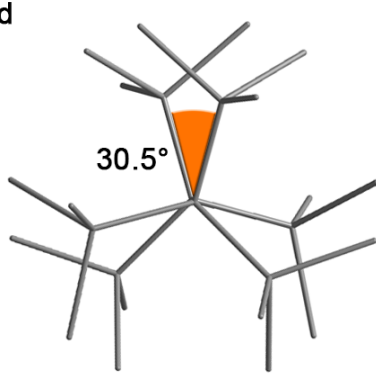

b

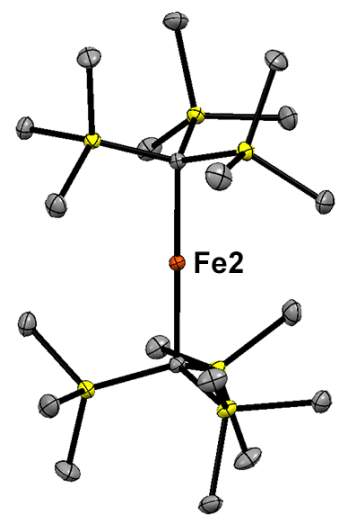

e

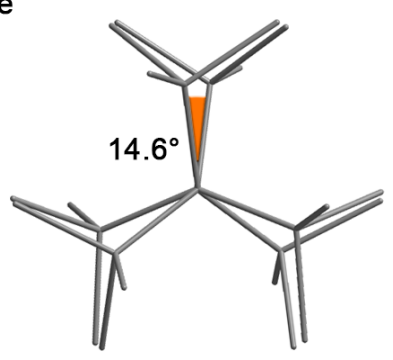

C

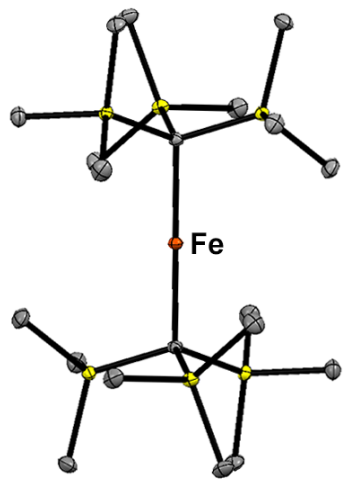

f

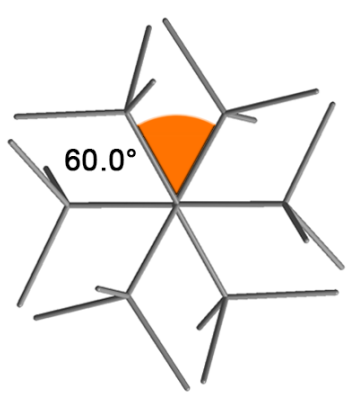


Figure 1. Structural data for compounds 1 and 2. a-c, Structures of the unique $\left[\mathrm{Fe}\left(\mathrm{C}\left(\mathrm{SiMe}_{3}\right)_{3}\right)_{2}\right]^{-}$complexes in the asymmetric unit of $\mathbf{1}(\mathbf{a}, \mathbf{b})$ and the $\mathrm{Fe}\left(\mathrm{C}\left(\mathrm{SiMe}_{3}\right)_{3}\right)_{2}$ molecular unit (twice the asymmetric unit) of $\mathbf{2}$ (c), as determined from the multipole models. Thermal ellipsoids are rendered at the $50 \%$ probability level. Grey, yellow, and orange ellipsoids represent $\mathrm{C}, \mathrm{Si}$, and $\mathrm{Fe}$ atoms, respectively; $\mathrm{H}$ atoms have been omitted for clarity. d-f, Wireframe structures of the two independent $\left[\mathrm{Fe}\left(\mathrm{C}\left(\mathrm{SiMe}_{3}\right)_{3}\right)_{2}\right]^{-}$moieties of $\mathbf{1}(\mathbf{d}, \mathbf{e})$ and the $\mathrm{Fe}\left(\mathrm{C}\left(\mathrm{SiMe}_{3}\right)_{3}\right)_{2}$ molecular unit (f), illustrating the dihedral angles between $\mathrm{Si}-\mathrm{C}-\mathrm{C}^{*}$ and $\mathrm{C}-\mathrm{C}^{*}-$ $\mathrm{Si}^{*}$ planes as described in the text (average values of $30.5^{\circ}, 14.6^{\circ}$, and $\sim 60.0^{\circ}$, respectively, are illustrated in d-f; carbon atoms are those bonded to iron). The Fe-C bonds in these structures are oriented roughly orthogonal to the plane of the text and hydrogen atoms are omitted for clarity. This figure was created using Olex $2^{35}$.

Laplacian and deformation electron density distributions. The Laplacian of the electron density, $\nabla^{2} \varrho$, is the sum of the diagonal elements of the Hessian matrix - obtained by taking the second derivative of $\varrho$ in the $x, y$, and $z$ directions - and is used to highlight local concentrations $\left(\nabla^{2} \varrho<0\right)$ or depletions $\left(\nabla^{2} \varrho>0\right)$ of electron density. Three-dimensional Laplacian distributions for 1 and 2 revealed six significantly negative minima, each located $\sim 0.32 \AA$ from each unique iron atom (i.e., in the iron $\mathrm{M}$-shell ${ }^{46}$ ). These minima roughly form an octahedron around each iron atom (Fig. 2a-c), with axial corners located along the $\mathrm{C}-\mathrm{Fe}-\mathrm{C}$ direction (i.e., close to the local $z$ axis on iron) and equatorial corners positioned near the plane orthogonal to this direction (i.e., close to the local iron $x y$ plane) ${ }^{47}$ Relative to the $\mathrm{C}-\mathrm{Fe}-\mathrm{C}$ direction, the octahedra are tilted slightly by $6-8^{\circ}$ (as seen in Fig. 2a-c). The Laplacian minima for each complex are quite distinct, as illustrated by two-dimensional Laplacian contour maps corresponding to selected planes containing an iron and four of the six minima (Fig. 2d-1). For compound 1, the four minima positioned close to the local iron $x y$ plane vary between -1084 and -965 e/ $\AA^{5}$ for Fe 1 and between -1128 and $-1083 \mathrm{e} / \AA^{5}$ for $\mathrm{Fe} 2$. The two minima near the $\mathrm{C}-\mathrm{Fe}-\mathrm{C}$ direction are -1045 
and $-1040 \mathrm{e} / \AA^{5}$ for $\mathrm{Fe} 1$ and $-1067 \mathrm{e} / \AA^{5}$ for Fe2. Thus, the four "equatorial" Laplacian minima are quite similar to the two "axial" minima in $\mathbf{1}$, differing by only $4-8 \%$ and indicating that the concentration of electron density is comparable along all three directions. In contrast, for $\mathbf{2}$ the four "equatorial" minima (values of -956 and $-1021 \mathrm{e} / \AA^{5}$ ) differ from the two "axial" minima $\left(-851 \mathrm{e} / \AA^{5}\right)$ by $12-19 \%$.
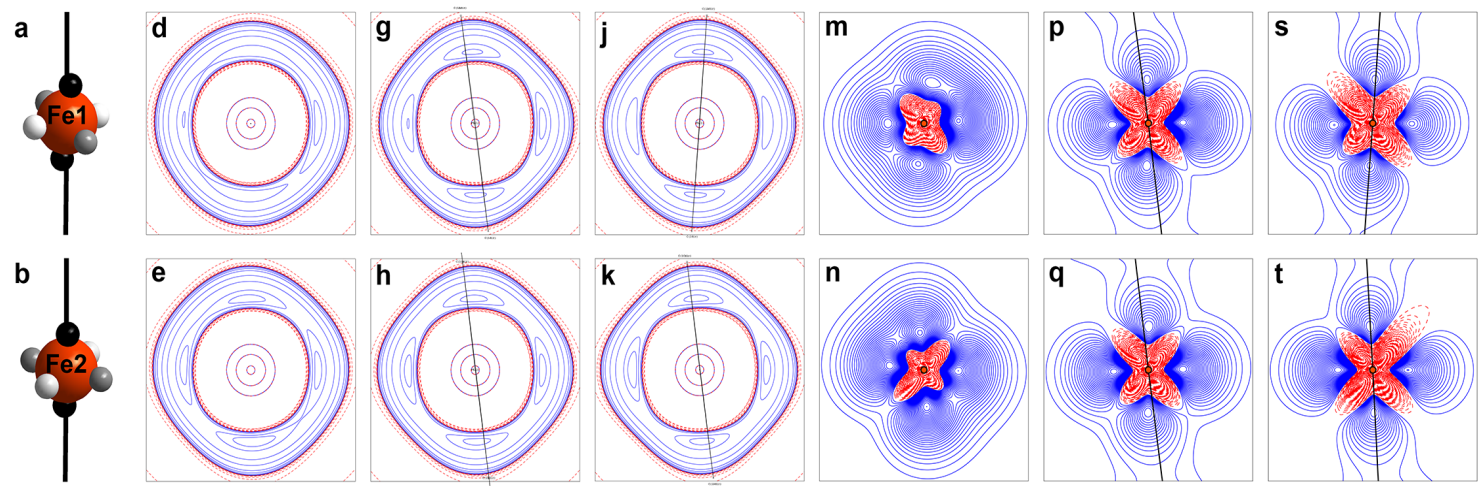

n
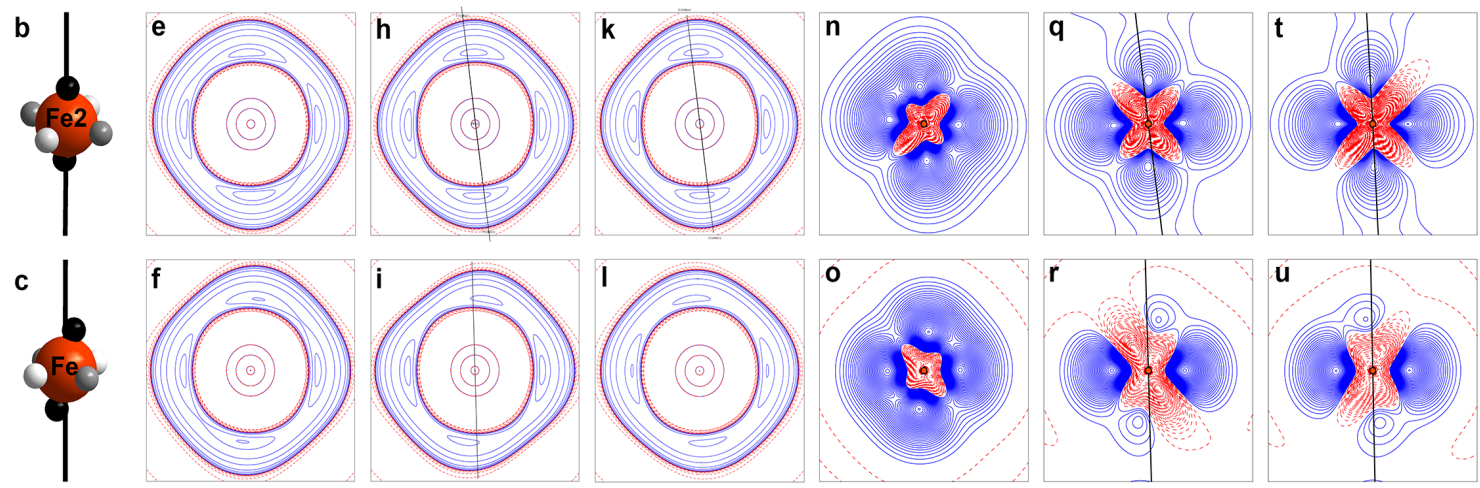

Figure 2 I Electron density distributions in 1 and 2. a-c, Locations of the Laplacian minima (charge concentrations) determined for the iron atoms in $\mathbf{1}(\mathbf{a}, \mathbf{b})$ and $\mathbf{2}(\mathbf{c})$ marked as white, gray, and black spheres. d-1, Laplacian contour maps with a width of $1 \AA$ for iron atoms in $\mathbf{1}$ and $\mathbf{2}$. $\mathbf{m}$ $\mathbf{u}$, Static model deformation electron density maps with a width of $2.2 \AA$ for iron atoms in $\mathbf{1}$ and 2. The planes in these maps are defined from the positions of the minima in a-c. The maps in the first column (d-f, m-o) include white and gray spheres, the maps in the second column (g-i, p-r) include the black and white spheres, and the maps in the third column (j-l, s-u) include the black and gray spheres. Solid blue lines represent positive contours of $-\nabla^{2} \varrho$ (local charge concentration) and the static model deformation electron density. Dashed red lines represent negative contours of $-\nabla^{2} \varrho$ (local charge depletion) and the static model deformation electron density. Laplacian contours are drawn at $\pm 2,4,8 \times 10^{n} \mathrm{e}^{-5}$, where $n$ is an integer ranging from -3 to +3 . An additional contour is drawn at $-\nabla^{2} \varrho=1000 \mathrm{e}^{-5}$ to emphasize the different 
numerical values of the Laplacian minima. The static model deformation electron density contours in $\mathbf{m}-\mathbf{u}$ are drawn in increments of $0.05 \mathrm{e}^{-3}$.

Contour maps of the static model deformation electron density surrounding the iron atoms in $\mathbf{1}$ and $\mathbf{2}$ are also depicted in Fig. $2 \mathrm{~m}-\mathrm{u}$, in the same planes as those presented for the Laplacian maps. The deformation density maps illustrate the difference between the multipole-expressed electron densities and hypothetical, independent atom model densities, wherein the atoms are represented by spherical isolated atom densities. Thus, these maps highlight regions where redistribution due to bonding has taken place. Within the framework of the ligand field model used here, the Laplacian and deformation density maps indicate a depletion of electron density in the $\mathrm{d}_{\mathrm{xz}}$ and the $\mathrm{d}_{\mathrm{yz}}$ orbitals for $\mathbf{1}$ and instead a preferential and similar filling of the $\mathrm{d}_{\mathrm{x}^{2}-\mathrm{y}}, \mathrm{d}_{\mathrm{xy}}$, and $d_{z^{2}}$ orbitals. For 2 , the maps indicate a preferential filling of the $d_{x^{2}-y^{2}}$ and $d_{x y}$ orbitals.

Experimental d-orbital occupancy estimates. Experimental d-orbital occupancies can be estimated from the multipole populations in the multipole model of the electron density, according to Holladay et $a l .^{29}$ (see the Experimental section for more details). It is important to emphasize that the angular component of the multipoles - and thus the subsequently derived dorbital occupancies - relate to user-defined local atomic coordinate systems and are therefore unrelated to the unit cell axes. Here, we define the local $z$ axis to point along the respective Fe-C directions for all iron atoms; however, for the $x$ and $y$ directions within the $x y$ plane, there is no clear optimal choice for these linear compounds. ${ }^{48}$ The extraction of d-orbital occupancies assumes that the electron density near the Fe site is primarily due to the atomic orbitals of Fe, i.e., that there is vanishing $\mathrm{Fe}-\mathrm{C}$ electronic overlap. Although this approximation is somewhat 
simplistic, it is justified in this scenario where the ligand field is weak for both $\mathbf{1}$ and $\mathbf{2}^{5,8}$ and the bonding between the ligands and the central $\mathrm{Fe}$ ion is primarily ionic in nature. The experimental d-orbital occupancies resulting from using this procedure are listed in Table 1 for the three unique Fe atoms in $\mathbf{1}$ and $\mathbf{2}$. The sum of the occupancies of the nearly degenerate $d_{x^{2}-y^{2}}$ and $d_{x y}$ orbitals is invariant with respect to rotation of the $x$ and $y$ axes around the $z$ axis, but the relative occupancies do depend on the orientation of these axes in the $x y$ plane. The same observation is true for the $\mathrm{d}_{\mathrm{xz}}$ and $\mathrm{d}_{\mathrm{yz}}$ orbitals. Thus, only the average occupancies are reported for these pairs in Table 1.

Table 1. Absolute values of the iron d-orbital occupancies obtained from the experimental multipole populations of $\mathbf{1}$ and $\mathbf{2}$; occupancy averages are reported for nearly degenerate $\left(\mathrm{d}_{\mathrm{xz}}, \mathrm{d}_{\mathrm{yz}}\right)$ and $\left(d_{x^{2}-y^{2}}, d_{x y}\right)$ pairs of orbitals. Percent occupancies of the valence electron count are given in italics.

\begin{tabular}{|c|c|c|c|}
\hline & $\left(\mathrm{d}_{\mathrm{xz}}, \mathrm{d}_{\mathrm{yz}}\right)$ & $\left(\mathrm{d}_{\mathrm{x}^{2}-\mathrm{y}^{2}}, \mathrm{~d}_{\mathrm{xy}}\right)$ & $\mathrm{d}_{\mathrm{z}^{2}}$ \\
\hline \multirow{2}{*}{$\mathrm{Fe} 1$} & $1.05(2)$ & $1.58(2)$ & $1.52(3)$ \\
\hline & $15.5(2) \%$ & $23.3(2) \%$ & $22.4(4) \%$ \\
\hline \multirow{2}{*}{$\mathrm{Fe} 2$} & $1.00(2)$ & $1.62(2)$ & $1.53(3)$ \\
\hline & $14.8(2) \%$ & $23.9(2) \%$ & $22.6(4) \%$ \\
\hline \multirow{2}{*}{$\mathrm{Fe}$} & $0.98(1)$ & $1.57(1)$ & $1.21(1)$ \\
\hline & $15.5(1) \%$ & $24.9(1) \%$ & $19.2(2) \%$ \\
\hline
\end{tabular}

The d-orbital occupancy estimates from the multipole populations do not provide direct information about the quantitative ordering of the d-orbital energies. However, when viewed in conjunction with wavefunction-based $a b$ initio calculations, the occupancies provide evidence 
that the Aufbau-principle is fulfilled for both $\mathbf{1}$ and $\mathbf{2}$ (see the Supplementary Information for complete details), which gives rise to the expected non-relativistic ${ }^{4,5} \mathrm{E}$ ground states. Hence, for compound 1 the d-orbital occupancies clearly support a model that requires that the $\mathrm{d}_{\mathrm{z}^{2}}$ orbital be significantly stabilized relative to the $\mathrm{d}_{\mathrm{xz}}$ and $\mathrm{d}_{\mathrm{yz}}$ orbitals, in contrast to their anticipated relative energies based on crystal field theory. Interestingly, although the two $\left[\mathrm{Fe}\left(\mathrm{C}\left(\mathrm{SiMe}_{3}\right)_{3}\right)_{2}\right]^{-}$moieties in 1 differ with respect to the relative orientation of their $\left[\mathrm{C}\left(\mathrm{SiMe}_{3}\right)_{3}\right]^{-}$ligands, their d-orbital occupancies are identical within a few standard deviations. For compound $\mathbf{2}$, the experimentallyderived occupancies suggest that the $d_{x^{2}-y^{2}}$ and $d_{x y}$ orbitals are lowest in energy followed by the $\mathrm{d}_{\mathrm{z}^{2}}, \mathrm{~d}_{\mathrm{xz}}$, and $\mathrm{d}_{\mathrm{yz}}$ orbitals, which are similar in energy.

Theoretical d-orbital occupancies. As mentioned above, theoretical studies have shown that the existence of magnetic blocking in $\mathbf{1}$ and $\mathbf{2}$ can be explained by the large differences in their relative d-orbital energies. ${ }^{8,12}$ In those studies, the d-orbital energies and their occupancies were derived using a ligand field analysis of ab initio results, in contrast to the experimental d-orbital occupancies derived in this work from a multipole-based reconstruction of the total electron density. The predicted d-orbital occupancies derived by the computational methods of population analysis are not necessarily quantitatively comparable to the experimental results derived here. To enable such a comparison, we used $a b$ initio calculations to compute structure factors representing the molecular electron density, and used these calculated values to refine multipole population parameters - again defining the local $z$ axis along the $\mathrm{Fe}-\mathrm{C}$ directions for all iron atoms. In this manner, we obtained theoretical d-orbital occupancies (Table 2) that are directly comparable to the experimental occupancies given in Table 1. The details of the structure factor calculation and subsequent multipole modeling from $a b$ initio calculations are given in the 
Supplementary Information. These theoretical d-orbital occupancies clearly reflect the d-orbital energy ordering resulting from $a b$ initio ligand field theory approaches, as presented in the original publications, ${ }^{6,19}$ suggesting that the procedure used here to extract d-orbital occupancies from a multipole expansion of the molecular electron density is highly reliable and a viable alternative to $a b$ initio ligand field theory analysis.

Table 2 | Absolute values of the iron d-orbital occupancies obtained from the theoretical multipole populations of $\mathbf{1}$ and $\mathbf{2}$; occupancy averages are reported for the nearly degenerate $\left(\mathrm{d}_{\mathrm{xz}}\right.$, $\left.d_{y z}\right)$ and $\left(d_{x^{2}-y^{2}}, d_{x y}\right)$ pairs of orbitals. Percent occupancies of the valence electron count are given in italics. Theoretical structure factors were calculated for only one of the two independent molecules in $\mathbf{1}$.

$\begin{array}{llll} & \left(\mathrm{d}_{\mathrm{xz}}, \mathrm{d}_{\mathrm{yz}}\right) & \left(\mathrm{d}_{\mathrm{x}^{2}-\mathrm{y}^{2}}, \mathrm{~d}_{\mathrm{xy}}\right) & \mathrm{d}_{\mathrm{z}^{2}} \\ \text { Fe1 (1) } & 1.09 & 1.33 & 1.57 \\ & 17.0 \% & 20.7 \% & 24.6 \% \\ & 1.14 & 1.50 & 1.11 \\ \text { Fe (2) } & 17.8 \% & 23.5 \% & 17.4 \%\end{array}$

\section{Discussion}

A comparison of the experimental and theoretical d-orbital occupancies determined from multipole parameterization of the electron density is given in Fig. 3. Overall, the two methods are in reasonably good agreement and differ within error for compound $\mathbf{2}$. In contrast, the two approaches give slightly different values for $\mathbf{1}$ - here, the experimental occupancies are $2 \%$ 
smaller than the theoretical values for the $\mathrm{d}_{\mathrm{z}^{2}}$ and $\mathrm{d}_{\mathrm{xz}} / \mathrm{d}_{\mathrm{yz}}$ orbitals and $3 \%$ larger than the theoretical values for the $\mathrm{d}_{\mathrm{x}^{2}-\mathrm{y}^{2}} / \mathrm{d}_{\mathrm{xy}}$ orbitals. In other words, theory predicts a larger accumulation of electron density along the local $z$ axis than in the $x y$-plane in $\mathbf{1}$, whereas experimentally these regions are found to have very similar densities. Importantly, the $\mathrm{d}_{\mathrm{z}^{2}}$ orbital occupancy is found to be $3-7 \%$ higher in $\mathbf{1}$ than $\mathbf{2}$ using both approaches, and thus the experimental results support the previous finding from theory ${ }^{8,12}$ that the $\mathrm{d}_{\mathrm{z}^{2}}$ orbital is substantially more occupied in $\mathbf{1}$ than in 2.

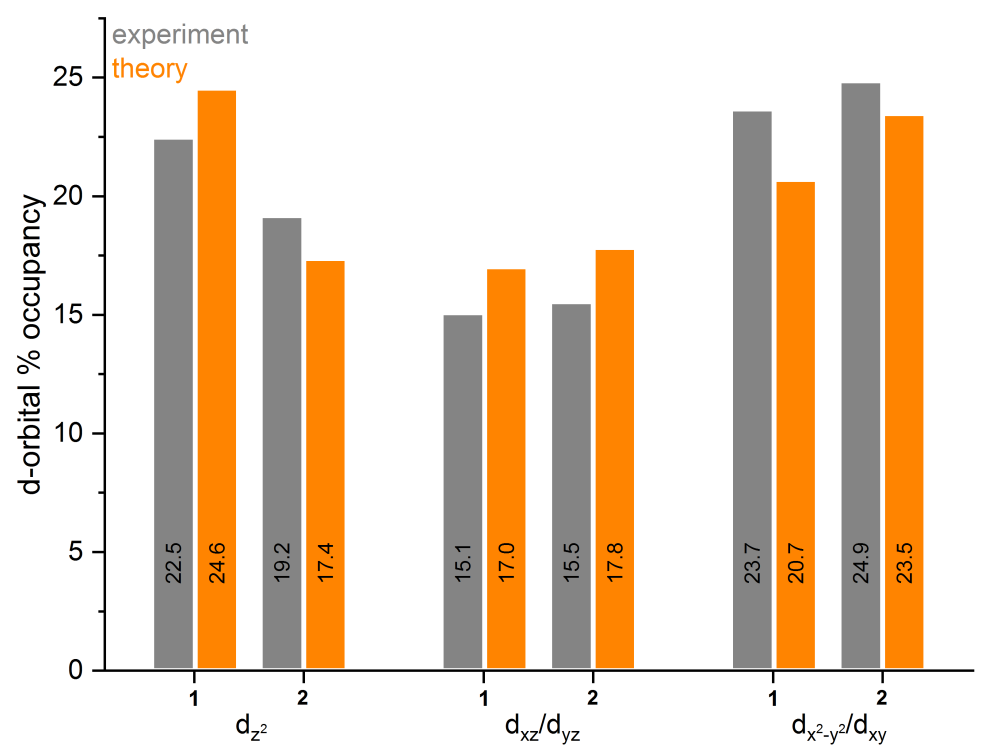

Figure 3 d-orbital occupancies from experiment and theory. Occupancies derived from multipole population parameters using experimental (grey) and theoretical (orange) structure factors. Occupancy averages (as percentages of the valence electron count) are reported for the nearly degenerate $\left(\mathrm{d}_{\mathrm{xz}}, \mathrm{d}_{\mathrm{yz}}\right)$ and $\left(\mathrm{d}_{\mathrm{x}^{2}-\mathrm{y}^{2}}, \mathrm{~d}_{\mathrm{xy}}\right)$ pairs of orbitals.

\section{Conclusion}

In summary, we have used single crystal X-ray diffraction data to determine the experimental electron density of two linear iron compounds $\left[\mathrm{Fe}\left(\mathrm{C}\left(\mathrm{SiMe}_{3}\right)_{3}\right)_{2}\right]^{-}$and $\mathrm{Fe}\left(\mathrm{C}\left(\mathrm{SiMe}_{3}\right)_{3}\right)_{2}$, and 
analyzed the data to reveal the existence of fundamentally different d-orbital occupancies in the two compounds. Spatial partitioning of the density also enabled demonstration of the different oxidation states and d-electron counts in both compounds. This combined data for each compound importantly provides experimental evidence for the origin of the large magnetic anisotropy in $\left[\mathrm{Fe}\left(\mathrm{C}\left(\mathrm{SiMe}_{3}\right)_{3}\right)_{2}\right]^{-}$and the corresponding exceptional slow magnetic relaxation behavior. An obvious extension of this method is to the study of electronic density distribution and magnetic anisotropy in lanthanide-based single-molecule magnets. Although the origin of magnetic anisotropy in these systems is different from that of transition metals, the asymmetry in 4f orbital populations arising from the ligand field will directly reveal the ground $M_{J}$ state. In addition, we will be able to quantify directly the degree of oblateness or prolateness of the valence electrons, which plays a particular role in the design of single-molecule magnets of the lanthanide ions. ${ }^{49}$ Work along these lines is underway.

\section{ASSOCIATED CONTENT}

\section{Supporting Information.}

The following files are available free of charge.

Details about data collection, data reduction, multipole modeling, theoretical calculations and theoretical structure factors. (PDF)

\section{AUTHOR INFORMATION}

\section{Corresponding Author}

jacobo@chem.au.dk, frank.neese@kofo.mpg.de, mihail.atanasov@kofo.mpg.de. 


\section{Author Contributions}

The manuscript was written through contributions of all authors. All authors have given approval to the final version of the manuscript.

\section{Funding Sources}

Danish National Research Foundation; U.S. National Science Foundation

\section{ACKNOWLEDGMENTS}

The Danish National Research Foundation is acknowledged for financial support (DNRF-93).

Danscatt is acknowledged for travel support. P.B. and J.R.L. were supported under NSF grant CHE-1464841. Mattia Sist, Emil Damgaard-Møller, Hidetaka Kasai, Espen Zink Eikeland and Jonas Beyer are thanked for their assistance collecting single crystal X-Ray data at the beamline BL02B1 at the SPring-8 synchrotron in Japan. We are grateful to Dr. Katie R. Meihaus for editorial assistance.

\section{REFERENCES}

1. Sessoli, R.; Gatteschi, D.; Caneschi, A.; Novak, M. A., Magnetic Bistability in a MetalIon Cluster. Nature 1993, 365 (6442), 141-143.

2. Woodruff, D. N.; Winpenny, R. E. P.; Layfield, R. A., Lanthanide Single-Molecule Magnets. Chem. Rev. 2013, 113 (7), 5110-5148.

3. Magnani, N.; Caciuffo, R., Future Directions for Transuranic Single Molecule Magnets. Inorganics 2018, 6 (1), 26.

4. $\quad$ Frost, J. M.; Harriman, K. L. M.; Murugesu, M., The rise of 3-d single-ion magnets in molecular magnetism: towards materials from molecules? Chem. Sci. 2016, 7 (4), 2470-2491. 5. Zadrozny, J. M.; Telser, J.; Long, J. R., Slow magnetic relaxation in the tetrahedral cobalt(II) complexes [Co(EPh)4]2- (EO, S, Se). Polyhedron 2013, 64 (0), 209-217.

6. Zadrozny, J. M.; Atanasov, M.; Bryan, A. M.; Lin, C. Y.; Rekken, B. D.; Power, P. P.; Neese, F.; Long, J. R., Slow magnetization dynamics in a series of two-coordinate iron(II) complexes. Chem. Sci. 2013, 4 (1), 125-138.

7. Zadrozny, J. M.; Long, J. R., Slow Magnetic Relaxation at Zero Field in the Tetrahedral Complex [Co(SPh)4]2-. J. Am. Chem. Soc. 2011, 133 (51), 20732-20734. 
8. Ishikawa, N.; Sugita, M.; Ishikawa, T.; Koshihara, S.; Kaizu, Y., Mononuclear lanthanide complexes with a long magnetization relaxation time at high temperatures: A new category of magnets at the single-molecular level. J. Phys. Chem. B 2004, 108 (31), 11265-11271.

9. Neese, F.; Pantazis, D. A., What is not required to make a single molecule magnet. Faraday Discuss. 2011, 148 (0), 229-238.

10. Ruiz, E.; Cirera, J.; Cano, J.; Alvarez, S.; Loose, C.; Kortus, J., Can large magnetic anisotropy and high spin really coexist? Chem. Commun. 2008, (1), 52-54.

11. Goodwin, C. A. P.; Ortu, F.; Reta, D.; Chilton, N. F.; Mills, D. P., Molecular magnetic hysteresis at 60 kelvin in dysprosocenium. Nature 2017, 548 (7668), 439-442.

12. Ding, Y.-S.; Chilton, N. F.; Winpenny, R. E. P.; Zheng, Y.-Z., On Approaching the Limit of Molecular Magnetic Anisotropy: A Near-Perfect Pentagonal Bipyramidal Dysprosium(III) Single-Molecule Magnet. Angew. Chem. Int. Ed. 2016, 55 (52), 16071-16074.

13. Gregson, M.; Chilton, N. F.; Ariciu, A.-M.; Tuna, F.; Crowe, I. F.; Lewis, W.; Blake, A. J.; Collison, D.; McInnes, E. J. L.; Winpenny, R. E. P.; Liddle, S. T., A monometallic lanthanide bis(methanediide) single molecule magnet with a large energy barrier and complex spin relaxation behaviour. Chem. Sci. 2016, 7 (1), 155-165.

14. Chilton, N. F.; Collison, D.; McInnes, E. J.; Winpenny, R. E.; Soncini, A., An electrostatic model for the determination of magnetic anisotropy in dysprosium complexes. Nat. Commun. 2013, 4, 2551.

15. Klahn, E. A.; Gao, C.; Gillon, B.; Gukasov, A.; Fabreges, X.; Piltz, R. O.; Jiang, S. D.; Overgaard, J., Mapping the Magnetic Anisotropy at the Atomic Scale in Dysprosium SingleMolecule Magnets. Chemistry 2018, 10.1002/chem.201803300.

16. Merrill, W. A.; Stich, T. A.; Brynda, M.; Yeagle, G. J.; Fettinger, J. C.; De Hont, R.; Reiff, W. M.; Schulz, C. E.; Britt, R. D.; Power, P. P., Direct Spectroscopic Observation of Large Quenching of First-Order Orbital Angular Momentum with Bending in Monomeric, TwoCoordinate Fe(II) Primary Amido Complexes and the Profound Magnetic Effects of the Absence of Jahn- and Renner-Teller Distortions in Rigorously Linear Coordination. J. Am. Chem. Soc. 2009, 131 (35), 12693-12702.

17. Reiff, W. M.; LaPointe, A. M.; Witten, E. H., Virtual Free Ion Magnetism and the Absence of Jahn-Teller Distortion in a Linear Two-Coordinate Complex of High-Spin Iron(II). $J$. Am. Chem. Soc. 2004, 126 (33), 10206-10207.

18. Reiff, W. M.; Schulz, C. E.; Whangbo, M. H.; Seo, J. I.; Lee, Y. S.; Potratz, G. R.; Spicer, C. W.; Girolami, G. S., Consequences of a Linear Two-Coordinate Geometry for the Orbital Magnetism and Jahn-Teller Distortion Behavior of the High Spin Iron(II) Complex $\mathrm{Fe}\left[\mathrm{N}(t-\mathrm{Bu})_{2}\right]_{2}$. J. Am. Chem. Soc. 2009, 131 (2), 404-405.

19. Atanasov, M.; Zadrozny, J. M.; Long, J. R.; Neese, F., A theoretical analysis of chemical bonding, vibronic coupling, and magnetic anisotropy in linear iron(ii) complexes with singlemolecule magnet behavior. Chem. Sci. 2013, 4 (1), 139-156.

20. LaPointe, A. M., Fe[C( $\left.\left.\mathrm{SiMe}_{3}\right)_{3}\right]_{2}$ : synthesis and reactivity of a monomeric homoleptic iron(II) alkyl complex. Inorg. Chim. Acta 2003, 345, 359-362.

21. Viefhaus, T.; Schwarz, W.; Hübler, K.; Locke, K.; Weidlein, J., Das unterschiedliche Reaktionsverhalten von basefreiem Tris(trimethylsilyl)methyl-Lithium gegenüber den Trihalogeniden der Erdmetalle und des Eisens. Z. Anorg. Allg. Chem. 2001, 627 (4), 715-725. 22. Zadrozny, J. M.; Xiao, D. J.; Atanasov, M.; Long, G. J.; Grandjean, F.; Neese, F.; Long, J. R., Magnetic blocking in a linear iron(I) complex. Nat. Chem. 2013, 5 (7), 577-581.

23. Gatteschi, D.; Sessoli, R.; Villain, J., Molecular Nanomagnets. 2006. 
24. Zadrozny, J. M.; Xiao, D. J.; Long, J. R.; Atanasov, M.; Neese, F.; Grandjean, F.; Long, G. J., Mössbauer Spectroscopy as a Probe of Magnetization Dynamics in the Linear Iron(I) and Iron(II) Complexes [Fe(C(SiMe3)3)2]1-/0. Inorg. Chem. 2013, 52 (22), 13123-13131.

25. Schwarz, W. H. E., The Full Story of the Electron Configurations of the Transition Elements. J. Chem. Educ. 2010, 87 (4), 444-448.

26. Atanasov, M.; Ganyushin, D.; Sivalingam, K.; Neese, F., A Modern First-Principles View on Ligand Field Theory Through the Eyes of Correlated Multireference Wavefunctions. In Molecular Electronic Structures of Transition Metal Complexes II, Mingos, D. M. P.; Day, P.; Dahl, J. P., Eds. Springer Berlin Heidelberg: Berlin, Heidelberg, 2012; pp 149-220.

27. Craven, M.; Nygaard, M. H.; Zadrozny, J. M.; Long, J. R.; Overgaard, J., Determination of d-Orbital Populations in a Cobalt(II) Single-Molecule Magnet Using Single-Crystal X-ray Diffraction. Inorg. Chem. 2018, 57 (12), 6913-6920.

28. Coppens, P., X-Ray Charge Densities and Chemical Bonding (IUCr Texts on Crystallography). IUCr: 1997; p 384.

29. Holladay, A.; Leung, P.; Coppens, P., Generalized Relations between D-Orbital Occupancies of Transition-Metal Atoms and Electron-Density Multipole Population Parameters from X-Ray-Diffraction Data. Acta Crystallogr. Sect. A 1983, 39 (May), 377-387.

30. CrysAlisPro, 1.171.37.31; Agilent Technologies: 2014.

31. RAPID-AUTO, Rigaku Corporation: Tokyo, Japan, 2004.

32. Blessing, R. H., Data Reduction and Error Analysis for Accurate Single Crystal Diffraction Intensities. Crystallography Reviews 1987, 1 (1), 3-58.

33. Blessing, R. H., DREADD - data reduction and error analysis for single-crystal diffractometer data. . J. Appl. Crystallogr. 1989, 22, 396-397.

34. Sheldrick, G., SHELXT - Integrated space-group and crystal-structure determination. Acta Crystallogr. Sect. A 2015, 71 (1), 3-8.

35. Dolomanov, O. V.; Bourhis, L. J.; Gildea, R. J.; Howard, J. A. K.; Puschmann, H., OLEX2: a complete structure solution, refinement and analysis program. J. Appl. Crystallogr. 2009, 42, 339-341.

36. Sheldrick, G. M., A short history of SHELX. Acta Crystallogr. Sect. A 2008, 64 (Pt 1), 112-22.

37. Volkov, A.; Macchi, P.; Farrugia, L. J.; Gatti, C.; Mallinson, P. R.; Richter, T.; Koritsanszky, T. XD2006 - a computer program for multipole refinement, topological analysis and evaluation of intermolecular energies from experimental and theoretical structure factors., revision 5.34; University at Buffalo, State University of New York, Buffalo, NY, University of Milano, Milano, Italy, University of Glasgow, Glasgow, U.K., CNRISTM, Milano, Italy, and Middle Tennessee State University Murfreesboro, TN, 2006.

38. Hansen, N. K.; Coppens, P., Electron Population Analysis of Accurate Diffraction Data .6. Testing Aspherical Atom Refinements on Small-Molecule Data Sets. Acta Crystallogr. Sect. A 1978, 34, 909-921.

39. Bader, R. F. W., Atoms in Molecules : A Quantum Theory. Clarendon Press: Oxford, 1990; p xviii, 438 p.

40. Neese, F., Software update: the ORCA program system, version 4.0. Wiley Interdisciplinary Reviews: Computational Molecular Science 2018, 8 (1), e1327.

41. Neese, F., The ORCA program system. Wiley Interdisciplinary Reviews: Computational Molecular Science 2012, 2 (1), 73-78. 
42. Zadrozny, J. M.; Xiao, D. J.; Atanasov, M.; Long, G. J.; Grandjean, F.; Neese, F.; Long, J. R., Magnetic blocking in a linear iron(I) complex. Nat Chem 2013, 5 (7), 577-581.

43. Farrugia, L. J.; Evans, C.; Senn, H. M.; Hanninen, M. M.; Sillanpaa, R., QTAIM View of Metal-Metal Bonding in Di- and Trinuclear Disulfido Carbonyl Clusters. Organometallics 2012, 31 (7), 2559-2570.

44. Schmokel, M. S.; Bjerg, L.; Cenedese, S.; Jorgensen, M. R. V.; Chen, Y.-S.; Overgaard, J.; Iversen, B. B., Atomic properties and chemical bonding in the pyrite and marcasite polymorphs of FeS2: a combined experimental and theoretical electron density study. Chem. Sci. 2014, $5,1408-1421$.

45. Schmokel, M. S.; Bjerg, L.; Overgaard, J.; Larsen, F. K.; Madsen, G. K.; Sugimoto, K.; Takata, M.; Iversen, B. B., Pushing X-ray electron densities to the limit: thermoelectric CoSb3. Angew. Chem. Int. Ed. 2013, 52 (5), 1503-6.

46. Shi, Z.; Boyd, R. J., The shell structure of atoms and the Laplacian of the charge density. J. Chem. Phys. 1988, 88 (7), 4375-4377.

47. In the case of exact cylindrical symmetry, there would obviously not be any extrema in the Laplacian (or the density) in the x-y plane. The extrema that we observe result from the influence of deviations from the exact symmetry.

48. It is obvious to choose the minima in the Laplacian distribution in the xy plane as defining the directions, but this does not minimize the cross terms which otherwise is a good indicator for a good choice of axes. In addition, the sum of the populations in the $\mathrm{dxy}$ and $\mathrm{dx2}$ $y 2$, and in the dxz and dyz, are constant upon rotation of the $x-y$ axes around the $z$-direction. We will therefore in the following use the sum of these, instead of individual d-orbital populations. 49. Rinehart, J. D.; Long, J. R., Exploiting single-ion anisotropy in the design of f-element single-molecule magnets. Chem. Sci. 2011, 2 (11), 2078-2085. 
For Table of Contents Only

High-resolution single-crystal X-ray diffraction studies have been employed to obtain experimental electron density distributions in the two well-known linear Fe-complexes $\left[\mathrm{Fe}\left(\mathrm{C}\left(\mathrm{SiMe}_{3}\right)_{3}\right)_{2}\right]^{0 / 1-}$. We unambiguously find a higher population of the $\mathrm{d}_{\mathrm{z}^{2}}$ orbital in the anion than in the neutral compound, validating the claim that the large magnetic anisotropy in both compounds arises from the presence of orbital angular momentum, despite the different oxidation state found in the two compounds.

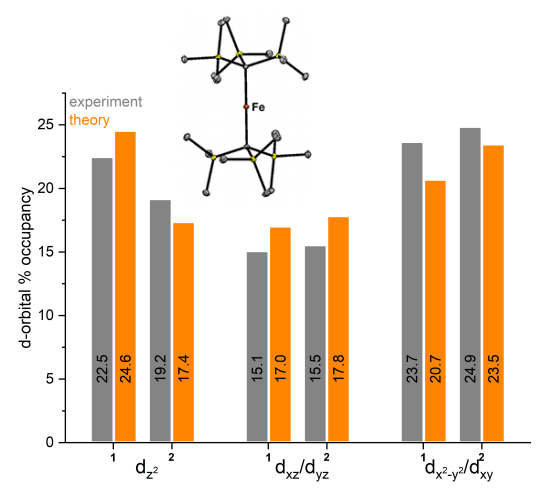

